\title{
COVID-19 Serological Tests: How well do they actually perform?
}

\author{
Abdi Ghaffari ${ }^{1,2}$, Robyn Meurant ${ }^{3}$, and Ali Ardakani ${ }^{1}$
}

1. Novateur Ventures Inc., Vancouver, BC CANADA

2. Department of Pathology and Molecular Medicine, Queen's University, Kingston, ON, Canada

3. NSF Health Sciences, Kirkbymoorside, York, United Kingdom

Corresponding authors:

Abdi Ghaffari (ghaffari@queensu.ca), Ali Ardakani (ali@novateur.org)

Novateur Ventures Inc.

1055 W Georgia Street, Suite 2100

Vancouver, BC, V6E 3P3

Canada

$\mathrm{T}:+1-844-200-6682$

ABBREVIATIONS

COVID-19, Coronavirus Disease 2019

SARS, Severe Acute Respiratory Syndrome

SARS-CoV, Severe Acute Respiratory Syndrome Coronavirus

SARS-CoV-2, Severe Acute Respiratory Syndrome Coronavirus 2

MERS, Middle East Respiratory Syndrome

BAL, Bronchoalveolar Lavage

EUA, Emergency Use Authorization

RT-PCR, Reverse Transcriptase Polymerase Chain Reaction

S, SARS-CoV-2 Spike protein 
E, SARS-CoV-2 Envelope protein

M, SARS-CoV-2 Membrane protein

NP, SARS-CoV-2 Nucleocapsid protein

RBD, Receptor-Binding Domain

ELISA, Enzyme-Linked Immunosorbent Assay

RDT, Rapid Diagnostic Test

CLIA, Chemiluminescence Immunoassay

LFIA, Lateral Flow Immunoassay

PoC, Point-of-Care

DABA, Double Antigen Bridging Assay 


\section{ABSTRACT}

In only a few months after initial discovery in Wuhan, China, SARS-CoV-2 and the associated COVID-19 disease has become a global pandemic causing significant mortality and morbidity. In the absence of vaccines and effective therapeutics, reliable serological testing can be a key element of public health policy to control further spread of the disease and gradually ease quarantine measures. However, prior to launch of large-scale seroprevalence studies to assess herd immunity, it is critical to understand the limits and potential of current SARS-CoV-2 serological tests on the market. In this study, we provide an overview of serological testing and conduct a systematic review of independent evaluations of SARS-CoV-2 serological tests performance. Our findings show significant variability in the accuracy of marketed tests and highlight several lab-based and point-of-care rapid diagnostic tests with high performance level in detecting SRAS-CoV-2 specific antibodies. The findings of this review highlight the need for ongoing independent evaluations of commercialized COVID-19 diagnostic tests.

\section{A. BACKGROUND}

Coronavirus disease 2019 (COVID-19) first was discovered as a cluster of patients with severe respiratory symptoms in Hubei Province, China, in December 2019. The early nucleic acid analysis of known pathogen panels led to negative results, suggesting the causative agent was of unknown origin. By early January 2020, analysis of bronchoalveolar lavage (BAL) fluid from infected patients revealed a pathogen, later named SARS-CoV-2, with 50\%, 80\%, and 96\% genetic sequence overlap to the genome of Middle East respiratory syndrome virus (MERSCoV), severe acute respiratory syndrome virus (SARS-CoV), and bat coronavirus RaTG13, 
respectively ${ }^{[1,2]}$. Like SARS-CoV and MERS-CoV, SARS-CoV-2 is a single-stranded RNA virus belonging to the beta genus Coronavirus in the Coronaviridae family ${ }^{[3]}$.

As SARS-CoV-2 can be transmitted from human to human, the disease has spread swiftly to over 200 countries, infecting nearly 6 million people, and resulting in at least 350,000 deaths worldwide (as of May 27, 2020 ${ }^{[4]}$. An unprecedented and rapidly growing global effort is underway for the development of COVID-19 vaccines and therapeutics, but at the time of this review there are no vaccines and only one antiviral drug (Remdesivir) approved under the FDA Emergency Use Authorization (EUA), with modest clinical benefit ${ }^{[5,6]}$. Under these circumstances, countries were forced to implement physical distancing measures to control the outbreak and in the process place approximately 3 billion people under lockdown.

\section{B. COVID-19 DIAGNOSTIC TESTS}

In any infectious disease outbreak, accurate and accessible diagnostic testing must be one of the pillars of control measure policies to understand the spread of disease and minimize it. The epidemiological studies of the outbreak in China estimated the proportion of undetected COVID-19 cases to be as high as $86 \%^{[7]}$. As asymptomatic or mild cases could play a significant role in the transmission and spread of the SARS-CoV-2 virus ${ }^{[7,8]}$, symptoms alone are not reliable diagnostic markers. There are two major types of diagnostic technologies available to address this: molecular and serological tests. Currently, much of the focus is on the SARS-CoV-2 molecular test, which can detect, with high accuracy, the viral-specific RNA molecules circulating in the host body. The gold standard molecular test is based on reverse-transcriptase- 
polymerase chain reaction (RT-PCR) technology. However, the PCR test is not useful in distinguishing between highly infective viruses versus ones that have been neutralized by the host, and it cannot assess immunity status against SARS-CoV-2 ${ }^{[9]}$. Serological-based antibody tests can complement molecular-based tests in providing a more accurate estimate of SARSCoV-2 incidence and potentially detect individuals with immunity against the disease as these tests detect markers of the immune response.

\section{HUMORAL IMMUNE RESPONSE TO SARS-COV-2}

In humoral immune response to infection, pathogen-specific antibodies, produced by B cells, neutralize and prevent further spread of the disease. The activation and differentiation of $B$ cells into antibody-secreting plasma B cells are triggered by a cascade of events involving virus digestion by antigen-presenting cells (e.g. dendritic cells, macrophages) and presentation of virus-specific antigen(s) to helper T cells (Fig 1). Antibodies protect the host by binding to specific antigens (proteins) on the virus to neutralize its fusion and entry into the host cell and facilitate recognition and killing by phagocytic immune cells ${ }^{[10]}$. In humans, three types of antibodies or immunoglobulins have been the target for COVID-19 serological tests: IgM, IgG, and IgA. Although the dynamics of the immune response in COVID-19 are not fully understood, typically IgM antibody is produced by host immune cells during the early stages of a viral infection. IgG is often the most abundant antibody in the blood and plays a more prominent role in the later stages of infection and in establishing long-term immune memory ${ }^{[11]}$. While IgM and IgG antibodies have been the leading candidates in COVID-19 serological test 
development, recent studies show that IgA, predominately present in the mucosal tissue, may also play a critical role in the immune response and disease progression ${ }^{[12]}$.

\section{SEROLOGICAL ANTIBODY TEST}

Serological or antibody tests detect immunoglobulins produced by the host's plasma B cells following exposure to foreign antigens. The SARS-CoV-2 genome encodes approximately 25 proteins that are required for infection and replication, including four major structural proteins: spike (S), envelope (E), membrane (M), and nucleocapsid (N) (Fig 1). The S protein plays a critical role in fusion and entry into the host cell and it comprises an $\mathrm{N}$-terminal S1 subunit (RBD and NTD domains) and a C-terminal S2 subunit. The primary function of the SARS-CoV-2 N protein (NP) is binding to the viral RNA genome and packing them into a helical nucleocapsid structure during viral replication ${ }^{[13,14]}$. Studies on the serum of recovered COVID-19 patients suggest that host neutralizing antibodies are primarily against $\mathrm{S}$ and $\mathrm{N}$ proteins ${ }^{[15,16]}$. Consequently, the likelihood of predicting immunity status could increase in serological tests that target various regions of $\mathrm{S}$ or $\mathrm{N}$ proteins. Therefore, the characterization of specific SARSCoV-2 antigen domains targeted by the humoral immune response becomes an integral part of the serological test development.

There are four major types of serological diagnostic tests: rapid diagnostic test (RDT), enzymelinked immunosorbent assay (ELISA), chemiluminescence immunoassay (CLIA), and neutralization assay. The neutralization assay is a lab-based test that uses live virus and cell 
culture methods to determine if patient antibodies can prevent viral infection in vitro. This test must be performed in laboratories with designated biosafety certificates to culture SARS-CoV-2infected cells and has a time-to-result of 3-5 days. RDT is a simple and rapid test based on lateral flow immunoassay (LFIA) technology, commonly found in pregnancy test kits for example. RDT can potentially be administered as a point-of-care (POC) or self-test. Typically, RDT test strips use a drop of blood to detect the presence of patient antibodies (IgG, IgM, or IgA) produced against a specific SARS-CoV-2 antigen (Fig 2A). RDT test is simple to use with time-to-result anywhere between 10-30 minutes. Therefore, it has the potential to be deployed in large-scale serological surveys. ELISA assay, currently the most commonly used format of the serological test, is a lab-based test with an average time-to-results of 2-5 hours. ELISA typically uses a surface coated with specific viral antigen(s), to bind and detect the corresponding patient antibodies ( $\lg G, \lg M, \lg A)$ in blood, plasma, or serum samples. The bound antigenantibody complex is then detected by using a second antibody and a substrate that produces a color or fluorescent-based signal. ELISA assays can be found in different formats including direct, competitive, and the most commonly used sandwich or double antigen bridging assay (DABA) (Fig 2B). CLIA technology follows a similar concept to ELISA by taking advantage of high binding affinity between the viral antigen(s) and host antibodies but uses chemical probes that yield light emission through a chemical reaction to generate a positive signal. CLIA has an average time-to-result of 1-2 hours. CLIA and ELISA are both suitable for large throughput labbased analysis and show good analytical agreement ${ }^{[17,18]}$.

\section{E. TIME KINETICS OF ANTIBODY RESPONSE IN COVID-19}


Knowledge of virus and host immune response dynamics are essential in formulating diagnostic test and treatment strategies. Studies of COVID-19 disease suggest that seroconversion, when antibody levels become detectable in the blood, may take place days after the viral load has peaked $^{[19]}$. Therefore, serological tests would be less effective in the early stages of COVID-19 disease. Wolfel and colleagues further confirmed these findings by reporting IgM and IgG seroconversion in $50 \%$ of the patients at one week after the onset of symptoms ${ }^{[20]}$. The median time for the detection of IgM and IgG in COVID-19 patients was reported to be 5 and 14 days, respectively $^{[21]}$. Yu and colleagues detected the seroconversion of IgA on day 2 and IgM/IgG on day 5 post onset of symptoms. Furthermore, the study reported $100 \%$ of cases had detectable levels of IgA, IgM, and IgG on day 32 post onset of symptoms ${ }^{[12]}$. Their findings also revealed that IgM and IgG levels to be significantly higher in severe COVID-19 cases compared to patients with the mild or moderate disease ${ }^{[12]}$, suggesting that serological tests require high sensitivity in detecting lower levels of antibodies in mild cases. Studies on the persistence of antibodies in blood suggest that high levels of IgG are detectable for at least 49 days after the onset of symptoms, while IgM levels declined rapidly on day 35 post infection ${ }^{[22]}$. The diagram in Figure 3 depicts the relative timelines and peak levels for SARS-CoV-2 viral load relative to blood IgM, IgG, and IgA antibodies. Improved understanding of humoral antibody response time kinetics in COVID-19 is crucial in the correct application of serological tests.

\section{F. SEROLOGICAL TEST PERFORMANCE}

The urgent need for the development of serological diagnostic tests in response to the COVID19 outbreak has compelled the regulatory bodies to implement emergency use authorization 
programs to expedite the commercialization process of these tests. In light of this, independent and robust post-market evaluations of COVID-19 serological tests are needed to confirm manufacturers' performance claims. The basic measures of quantifying a diagnostic test performance are sensitivity and specificity. Sensitivity is the ability of a test to detect the disease agent or host response to the disease (i.e., antibodies) when it is truly present, whereas specificity is the probability of a test to correctly return a negative result when disease or host response is absent ${ }^{[23]}$.

In this review, we conducted a systematic review of independent studies that assessed the performance of currently available SARS-CoV-2 serological tests. We included studies that reported sensitivity and specificity, stage of disease (early, intermediate, and late), the test format (CLIA, ELISA, RDT), and target antibody target (IgA, $\lg G$, $\lg M$, or $\lg G+\lg M)^{[24,25]}$. Where possible, we recorded the SARS-CoV-2 antigens used for antibody detection, although this information was made readily available by all manufacturers. The studies that did not specify the disease stage of test samples were grouped under the "overall" category and assessed separately. In total, we reviewed performance data on 5 CLIA, 15 ELISA, and 42 RDT serological tests currently on the market (see Table S1 in supplementary file).

The distribution plot of the whole data shows a higher degree of variability in test sensitivity values compared to specificity (Fig 4). This level of variability further emphasizes the need for independent evaluations of serological tests on the market. The sensitivity/specificity plots highlight tests at various stages of COVID-19 disease and confirms the expectation that 
serological tests are more effective in later stages of the disease when higher IgG and IgM levels are present in the blood (Fig 5). The heatmap of tests in the "overall" category ranks the highest performing test in each target antibody category based on sensitivity followed by specificity (Fig 6). Top-performing COVID-19 serological tests (>95\% sensitivity and specificity) from xy plots and heatmap are summarized in Table 1.

Table 1. Top-performing COVID-19 serological tests based on independent evaluations of sensitivity/specificity.

\begin{tabular}{|c|c|c|c|c|c|c|c|c|}
\hline No. & Test Name ${ }^{[r e f]}$ & Manufacturer & Format & $\begin{array}{c}\text { Target } \\
\text { Antibody }\end{array}$ & $\begin{array}{l}\text { Disease } \\
\text { Stage }\end{array}$ & $\begin{array}{c}\text { Sensitivity } \\
(\%)\end{array}$ & $\begin{array}{c}\text { Specificity } \\
(\%)\end{array}$ & $\begin{array}{l}\text { SARS- } \\
\text { CoV-2 } \\
\text { Antigen }\end{array}$ \\
\hline 2 & $\begin{array}{l}\text { Wantai SARS-CoV- } \\
2 \mathrm{Ab} \text { ELISA }^{[26]}\end{array}$ & $\begin{array}{l}\text { Beijing Wantai } \\
\text { Biological } \\
\text { Pharmacy }\end{array}$ & ELISA & $\lg G / \lg M / \lg A$ & Overall & 95.4 & 100 & RBD \\
\hline 8 & $\begin{array}{l}2019-n C o V \operatorname{lgG} / \operatorname{lgM} \\
\text { Antibody Detection } \\
\mathrm{Kit}^{[27]}\end{array}$ & $\begin{array}{l}\text { Zhuhai Lizhu } \\
\text { Reagent }\end{array}$ & ELISA & $\lg G / \lg M$ & Late $(>14 d)$ & 95 & 100 & NP \\
\hline 6 & $\mathrm{CMIA-Ab^{[28] }}$ & $\begin{array}{l}\text { Xiamen InnoDx } \\
\text { Biotech Co., } \\
\text { Ltd. }\end{array}$ & CLIA & $\lg \mathrm{G} / \lg M$ & Overall & 96.2 & 99.3 & RBD \\
\hline 10 & $\begin{array}{l}\text { Chemiluminescence } \\
\text { detection } \mathrm{kit}^{[29]}\end{array}$ & $\begin{array}{l}\text { Shenzhen Yhlo } \\
\text { Biotech }\end{array}$ & CLIA & $\begin{array}{l}\lg M \\
\lg G\end{array}$ & Overall & $\begin{array}{l}100 \\
100 \\
\end{array}$ & $\begin{array}{l}98.5 \\
99 \\
\end{array}$ & $\mathrm{n} / \mathrm{a}$ \\
\hline 13 & $\begin{array}{l}\text { MAGLUMI 2019- } \\
\text { nCoV IgG }\end{array}$ & Snibe Co. & CLIA & $\lg G$ & Overall & 98.8 & 95.1 & $\mathrm{n} / \mathrm{a}$ \\
\hline 61 & $\begin{array}{l}\text { Architect SARS- } \\
\text { CoV-2 IgG Assay }{ }^{[30]}\end{array}$ & Abbott & CLIA & $\lg G$ & Late $(>14 d)$ & 97.2 & 100 & NP \\
\hline 5 & $\begin{array}{l}\text { Wantai SARS-CoV- } \\
2 \text { Ab Rapid Test }{ }^{[28]}\end{array}$ & $\begin{array}{l}\text { Beijing Wantai } \\
\text { Biological } \\
\text { Pharmacy }\end{array}$ & RDT & $\lg G / \lg M / \lg A$ & Overall & 97.5 & 95.2 & RBD \\
\hline 33 & $\begin{array}{l}\text { NG-Test IgM-IgG } \\
\text { COVID All-in-One } \\
\text { lateral flow } \\
\text { immunoassay }^{[25]}\end{array}$ & NG Biotech & RDT & $\lg \mathrm{G} / \lg M$ & Late $(>14 d)$ & 97 & 100 & $\mathrm{n} / \mathrm{a}$ \\
\hline 34 & $\begin{array}{l}\text { Covid-19 lgG/lgM } \\
\text { Antibody Test }{ }^{[25]}\end{array}$ & Biomerica & RDT & $\lg G$ & $\begin{array}{l}\text { Intermediate } \\
(8-14 d)\end{array}$ & 100 & 100 & $\mathrm{n} / \mathrm{a}$ \\
\hline 35 & $\begin{array}{l}\text { Acro Biotech } \\
\text { COVID-19 Rapid } \\
\text { POC test }\end{array}$ & Acro Biotech & RDT & $\lg G$ & $\begin{array}{l}\text { Intermediate } \\
(8-14 d)\end{array}$ & 100 & 100 & $\mathrm{n} / \mathrm{a}$ \\
\hline 37 & $\begin{array}{l}\text { DiagnoSure COVID- } \\
19 \text { lgG/lgM rapid } \\
\text { test cassette } \\
\end{array}$ & $\begin{array}{l}\text { GritOverseas } \\
\text { Pte. }\end{array}$ & RDT & $\begin{array}{l}\lg G \\
\lg M\end{array}$ & $\begin{array}{l}\text { Intermediate } \\
(8-14 d)\end{array}$ & $\begin{array}{l}100 \\
100\end{array}$ & $\begin{array}{l}100 \\
100\end{array}$ & $\mathrm{n} / \mathrm{a}$ \\
\hline 41 & $\begin{array}{c}\text { Rapid SARS-CoV-2 } \\
\text { Antibody } \\
(\operatorname{lgM} / \operatorname{lgG})^{[25]}\end{array}$ & $\begin{array}{l}\text { AMEDA } \\
\text { Labordiagnostik } \\
\text { GmbH }\end{array}$ & RDT & $\lg G / \lg M$ & Late $(>14 d)$ & 95.7 & 97.4 & $\mathrm{n} / \mathrm{a}$ \\
\hline 57 & $\begin{array}{l}\text { COVID-19 lgG/lgM } \\
\text { Rapid Test } \\
\text { Cassette }^{[24]}\end{array}$ & Healgen & RDT & $\begin{array}{l}\lg M \\
\lg G\end{array}$ & Overall & $\begin{array}{l}100 \\
96.7\end{array}$ & $\begin{array}{l}100 \\
97.5\end{array}$ & $\mathrm{n} / \mathrm{a}$ \\
\hline
\end{tabular}

\section{G. SEROPREVALENCE OF SARS-COV-2 SPECIFIC ANTIBODIES}


In light of policies to ease the lockdown and re-open the economy, large-scale seroprevalence studies to screen for immunity status are being implemented in several jurisdictions. Critics point to gaps in our understanding of immune response to COVID-19 infection including the ability of serological tests in detecting neutralizing antibodies and the capacity of the immune system in providing long-term immunity against SARS-CoV-2. However, some argue that in the context of a global viral outbreak with a relatively high mortality rate, inaction due to uncertainty can have negative consequences compared to the harm caused by serological test false positive and false negative results ${ }^{[31]}$. Several jurisdictions have initiated seroprevalence studies to provide a more accurate estimate of cases with positive SARS-CoV-2-specific antibodies, irrespective of disease symptoms. In Los Angeles County, the prevalence of SARSCoV-2 antibodies in the community was estimated to be $4.65 \%$, equivalent to 367,000 adults, which was substantially greater than 8430 confirmed cases in the same county at the time of the study ${ }^{[32]}$. In New York City, $19.9 \%$ of the population has been estimated to have SARS-CoV-2 antibodies, compared to $2.1 \%$ confirmed cases as of May 2, $2020^{[33]}$. Similar studies from Germany, UK, Singapore, and China show significantly higher estimates of positive SARS-CoV-2 antibody cases compared to symptomatic cases confirmed by molecular tests ${ }^{[34]}$. As undetected cases, with mild or no symptoms, can transmit the virus, it is not surprising that countries (e.g., South Korea, Germany, or Singapore) with large-scale and well-organized testing program, combined with extensive isolation and contact tracing of infected, have had some success in minimizing COVID-19 related death in their population ${ }^{[35]}$.

\section{H. CONCLUDING REMARKS}


As serology tests are in high demand, in part due to an increase in large-scale seroprevalence studies, it is imperative for the national and regional governments to continue coordinated efforts to independently validate serology tests performance and also partner with industry to scale-up manufacturing and production capacity. Also, existing emergency authorization programs, intended to accelerate the manufacturing of diagnostic tests, must be accompanied by informed and clear guidelines on preferred and minimally acceptable profiles of the COVID19 serological test designed for a specific indication.

Despite the unprecedented response to the outbreak, there remain major gaps in our understanding of the interaction between SARS-CoV-2 and the immune system, which can negatively impact the utilization of serological testing. Coordinated research efforts are urgently needed to investigate some of the key gaps in our knowledge, including:

1. Which serological tests can identify SARS-CoV-2 neutralizing antibodies?

2. Is there cross-reactivity between neutralizing antibodies to other coronaviruses?

3. What are optimal SARS-CoV-2 antigens for the detection of neutralizing antibodies?

4. What is the correlation between SARS-CoV-2-specific antibodies and protective immunity status?

5. How long does protective immunity last in recovered patients? Are individuals susceptible to re-infection with SARS-CoV-2?

6. Is humoral antibody response the best indicator for protective immunity or are there other immune cell-based mechanisms?

In the context of the COVID-19 outbreak and execution of return-to-work policies, failing to take advantage of available diagnostic tools due to uncertainty can have profound consequences. Medical professionals frequently rely on imperfect evidence with the possibility of false positives and false negatives. It is however important to clearly understand the limits and potentials of serological tests to make informed decisions based on risk and benefit assessment in each specific situation. In the words of Tedros Adhanom Ghebreyesus, director- 
general of the World Health Organization "countries cannot fight this pandemic blindfolded.

Countries should know where the cases are." 


\section{Figure Legends}

Figure 1. The human antibody response to SARS-CoV-2 infection. (1) The SARS-CoV-2 virus enters the host cell via interaction between viral spike (S) and host Angiotensin-converting Enzyme-2 (ACE2) proteins. (2) Following replication and release from the host cells, a subset of viruses will be engulfed and digested by antigen-presenting cells (APC) like macrophages or dendritic cells (3). Fragmented SARS-CoV-2 antigen(s) will be presented to T helper cells, which in turn will interact and activate B cells (4). Activated B cells will proliferate and differentiate into plasma or memory B cells with a high-affinity binding receptor for the original SARS-CoV-2 antigen (5). Plasma cells secrete their SARS-CoV-2-specific receptors in the form of IgM, IgG, or IgA antibodies. Antibody-mediated neutralization occurs when SARS-CoV-2-specific antibodies bind to viral antigen(s) and prevent virus interaction and entry into host cells (6).

\section{Figure 2. Serological diagnostic tests.}

Panel A: Rapid diagnostic test (RDT) are typically based on a colorimetric lateral flow immunoassay, where host target antibodies (IgM, IgG, or IgA) within a few droplets of blood flow across an adhesive pad (e.g., nitrocellulose) and interact with bound virus-specific antigens and secondary antibodies (anti-human IgM/G antibodies). Conjugated SARS-CoV-2-specific antigen(s) (labeled with gold here), binds with the corresponding host antibodies. As antibodyantigen complexes travel up the membrane, bound anti-SARS-CoV-2 IgM interact with fixed anti-IgM secondary antibodies on the $M$ line and anti-SARS-CoV-2 IgG with anti-IgG on the $G$ line. If the blood sample does not contain SARS-CoV-2-specific antibodies, the M or G lines do not appear in the final test results, and only the control (C) line will be revealed. 
Panel B: Enzyme-linked immunosorbent assay (ELISA) can be presented in different formats based on differences in antigen immobilization and antibody labeling. In Direct ELISA, SARSCoV-2 antigen(s) attach to a plastic solid phase and detected by the addition of a conjugated antibody. In Sandwich ELISA, the capture antibody is attached to the plastic solid phase. Antigen(s) in the sample will bind to the capture antibody and detected by a second enzymelabeled antibody. In Competitive ELISA, sample SARS-CoV-2 antigen is pre-incubated with the primary antibody and then added to a well coated with a secondary antibody along with an enzyme-conjugated antigen that competes with sample antigen for binding with the primary antibody. The more SARS-CoV-2 antigen in the sample, the less conjugated antigen will be bound, and lower the signal.

Figure 3: Time kinetics of antibody response in COVID-19. illustration on the relative levels of host immunoglobulins (IgM, IgG, IgA) in relation to SARS-CoV-2 viral load and onset of symptoms. Specific antibody seroconversion occurs when it reaches the detectable level.

Disclaimer: for illustrative purposes only and does not represent actual levels of each antibody.

Figure 4. Overview of reported sensitivity and specificity of COVID-19 serological tests.

Reported performance data from categories were plotted based on target antibody (IgG, IgM, IgG/IgM) to provide an overview of variability distribution in marketed diagnostic tests. Blue bars represent the mean values. 
Figure 5. Independent evaluation of COVID-19 serological tests at various stages of the

disease. $\mathrm{XY}$ plots of reported sensitivity and specificity in detection of IgM, IgG, or IgG/IgM antibodies were generated at different stages of the disease include early (1-7 days post symptom onset), intermediate ( $7-14$ days post symptoms onset), and late ( $>14$ days post symptom onset). ELISA: enzyme-linked immunosorbent assay; CLIA: chemiluminescence immunoassay; RDT: rapid diagnostic test (lateral flow immunoassay). Refer to Figure 6 for test names and manufacturer corresponding to each number shown here.

Figure 6. Independent evaluation of SARS-CoV-2 serologic test overall performance.

Sensitivity and specificity data from studies that did not specify the COVID-19 disease stage ("overall" group) are represented in a heat map. The heatmap is ordered according to the antibody target (IgG, IgM, and IgG/IgM) followed by sensitivity and specificity values. All analyses were conducted using software R version 3.6.3. Heatmaps were generated using the gplots and RColorBrewer packages. ELISA: enzyme-linked immunosorbent assay; CLIA: chemiluminescence immunoassay; RDT: rapid diagnostic test. 
CONFLICT OF INTEREST STATEMENT

This study is sponsored by Novateur Ventures Inc., Vancouver, Canada. Authors declare no conflict of interest and no affiliation with manufactures named in this study. 


\section{References}

1. Zhu, N., et al., A Novel Coronavirus from Patients with Pneumonia in China, 2019. N Engl J Med, 2020. 382(8): p. 727-733. DOI: 10.1056/NEJMoa2001017

2. Zhou, P., et al., A pneumonia outbreak associated with a new coronavirus of probable bat origin. Nature, 2020. 579(7798): p. 270-273. DOI: 10.1038/s41586-020-2012-7

3. Lu, R., et al., Genomic characterisation and epidemiology of 2019 novel coronavirus: implications for virus origins and receptor binding. Lancet, 2020. 395(10224): p. 565-574. DOI: 10.1016/S0140-6736(20)30251-8

4. World Health Organization COVID-19 Situation Report 127. https://www.who.int/docs/default-source/coronaviruse/situation-reports (accessed May 27, 2020).

5. Wang, Y., et al., Remdesivir in adults with severe COVID-19: a randomised, double-blind, placebo-controlled, multicentre trial. Lancet, 2020. 395(10236): p. 1569-1578. DOI: $10.1016 / \mathrm{S} 0140-6736(20) 31022-9$

6. Grein, J., et al., Compassionate Use of Remdesivir for Patients with Severe Covid-19. N Engl J Med, 2020. DOI: 10.1056/NEJMoa2007016

7. Li, R., et al., Substantial undocumented infection facilitates the rapid dissemination of novel coronavirus (SARS-CoV-2). Science, 2020. 368(6490): p. 489-493. DOI:

10.1126/science.abb3221

8. Bai, Y., et al., Presumed Asymptomatic Carrier Transmission of COVID-19. JAMA, 2020. DOI: 10.1001/jama.2020.2565 
9. Winter, A.K. and S.T. Hegde, The important role of serology for COVID-19 control. Lancet Infect Dis, 2020. DOI: 10.1016/S1473-3099(20)30322-4

10. Janeway C.A., e.a., Immunobiology, 5th edition: The Immune System in Health and Disease. 2001: Garland Publishing.

11. Azkur, A.K., et al., Immune response to SARS-CoV-2 and mechanisms of immunopathological changes in COVID-19. Allergy, 2020. DOI: 10.1111/all.14364

12. $\mathrm{Yu}, \mathrm{H} . \mathrm{Q} .$, et al., Distinct features of SARS-CoV-2-specific IgA response in COVID-19 patients. Eur Respir J, 2020. DOI: 10.1183/13993003.01526-2020

13. Parks, J.M. and J.C. Smith, How to Discover Antiviral Drugs Quickly. N Engl J Med, 2020. DOI: 10.1056/NEJMcibr2007042

14. Cong, Y., et al., Nucleocapsid Protein Recruitment to Replication-Transcription Complexes Plays a Crucial Role in Coronaviral Life Cycle. J Virol, 2020. 94(4). DOI: 10.1128/JVI.01925-19

15. Ni, L., et al., Detection of SARS-CoV-2-Specific Humoral and Cellular Immunity in COVID19 Convalescent Individuals. Immunity, 2020. DOI: 10.1016/j.immuni.2020.04.023

16. Jiang, S., et al., Neutralizing Antibodies against SARS-CoV-2 and Other Human Coronaviruses: (Trends in Immunology 41, 355-359; 2020). Trends Immunol, 2020. DOI: 10.1016/j.it.2020.04.008

17. Vashist, S.K., In Vitro Diagnostic Assays for COVID-19: Recent Advances and Emerging Trends. Diagnostics (Basel), 2020. 10(4). DOI: 10.3390/diagnostics10040202 
18. Yan, Y., et al., Laboratory testing of SARS-CoV, MERS-CoV, and SARS-CoV-2 (2019-nCoV): Current status, challenges, and countermeasures. Rev Med Virol, 2020: p. e2106. DOI: $10.1002 / \mathrm{rmv} .2106$

19. To, K.K., et al., Temporal profiles of viral load in posterior oropharyngeal saliva samples and serum antibody responses during infection by SARS-CoV-2: an observational cohort study. Lancet Infect Dis, 2020. 20(5): p. 565-574. DOI: 10.1016/S1473-3099(20)30196-1

20. Wolfel, R., et al., Virological assessment of hospitalized patients with COVID-2019. Nature, 2020. DOI: 10.1038/s41586-020-2196-x

21. Guo, L., et al., Profiling Early Humoral Response to Diagnose Novel Coronavirus Disease (COVID-19). Clin Infect Dis, 2020. DOI: 10.1093/cid/ciaa310

22. Tan W, L.Y., Zhang J, et al., Viral Kinetics and Antibody Responses in Patients with COVID19. medRxiv (preprint), 2020. DOI: https://doi.org/10.1101/2020.03.24.20042382

23. Mandrekar, J.N., Simple statistical measures for diagnostic accuracy assessment. J Thorac Oncol, 2010. 5(6): p. 763-4. DOI: 10.1097/JTO.0b013e3181dab122

24. U.S. Food \& Drug Administratin. Independent Evaluations of COVID-19 Serological Tests. (Accessed June 6, 2020). https://open.fda.gov/apis/device/covid19serology/.

25. FINDDX - SARS-COV-2 DIAGNOSTICS: PERFORMANCE DATA (Accessed May 20, 2020). https://www.finddx.org/covid-19/dx-data/

26. Ria Lassaunière, A.F., Zitta B. Harboe, Alex C.Y. Nielsen, Anders Fomsgaard, Karen A. Krogfelt, Charlotte S. Jørgensen, Evaluation of nine commercial SARS-CoV-2 immunoassays. medRxiv (preprint), 2020. DOI: https://www.medrxiv.org/content/10.1101/2020.04.09.20056325v1 
27. Liu, W., et al., Evaluation of Nucleocapsid and Spike Protein-Based Enzyme-Linked Immunosorbent Assays for Detecting Antibodies against SARS-CoV-2. J Clin Microbiol, 2020. 58(6). DOI: 10.1128/JCM.00461-20

28. Lou, B., et al., Serology characteristics of SARS-CoV-2 infection since the exposure and post symptoms onset. medRxiv (preprint), 2020. DOI:

\section{https://doi.org/10.1101/2020.03.23.20041707}

29. Jin Zhang, J.L., Na Li, Yong Liu, Rui Ye, Xiaosong Qin, Rui Zheng, Serological detection of 2019-nCoV respond to the epidemic: A useful complement to nucleic acid testing.

medRxiv (preprint), 2020. DOI: https://doi.org/10.1101/2020.03.04.20030916

30. Bryan, A., et al., Performance Characteristics of the Abbott Architect SARS-CoV-2 IgG Assay and Seroprevalence in Boise, Idaho. J Clin Microbiol, 2020. DOI:

\subsection{8/JCM.00941-20}

31. Weinstein, M.C., et al., Waiting for Certainty on Covid-19 Antibody Tests - At What Cost? N Engl J Med, 2020. DOI: 10.1056/NEJMp2017739

32. Sood, N., et al., Seroprevalence of SARS-CoV-2-Specific Antibodies Among Adults in Los Angeles County, California, on April 10-11, 2020. JAMA, 2020. DOI:

10.1001/jama.2020.8279

33. New York State Governor Website (posted May 2, 2020).

https://www.governor.ny.gov/news/amid-ongoing-covid-19-pandemic-governorcuomo-announces-results-completed-antibody-testing. 
34. Popovich, N., , Sanger-Katz, M., The World Is Still Far From Herd Immunity for Coronavirus. https://www.nytimes.com/interactive/2020/05/28/upshot/coronavirusherd-immunity.html, May 28, 2020.

35. Cohen, J. and K. Kupferschmidt, Countries test tactics in 'war' against COVID-19. Science, 2020. 367(6484): p. 1287-1288. DOI: 10.1126/science.367.6484.1287 